\title{
A NEW STATE-LEVEL PANEL OF ANNUAL INEQUALITY MEASURES OVER THE PERIOD $1916-2005$
}

\author{
Mark W. Frank \\ Sam Houston State University • Huntsville, TX
}

\section{ABSTRACT}

This paper introduces a new panel of annual state-level income inequality measures over the ninety year period 1916-2005. Among many of the states inequality followed a U-shaped pattern over the past century, peaking both before the Great Depression and again at the time of the new millennium. The new panel reveals significant state-level variations, both before the year 1945, and regionally. While Northeastern states are strongly correlated with aggregate U.S. trends, we find many of the Western states have little overall correlation over the past century. The availability of this new panel may prove useful to empirical researchers interested in all aspects of income inequality, particularly given the panel's unusually large number of both time-series and cross-sectional observations

\section{INTRODUCTION}

As the threat of war loomed, the $16^{\text {th }}$ Amendment to the U.S. Constitution was ratified on February $3^{\text {rd }} 1913$, giving the U.S. Congress the authority to levy a federal income tax. ${ }^{1}$ Congress followed by adopting a $1 \%$ tax on incomes of more than $\$ 3,000$, with a surtax of $6 \%$ on incomes of more than $\$ 500,000$. Since 1916 , the Internal Revenue Service (IRS) has published income and tax statistics based on information reported on the Federal tax returns filed by U.S. individual taxpayers. These annual IRS publications provide unique and comprehensive access to the incomes of Americans over the past century.

The primary innovation of this paper is to use IRS income tax filing data to construct a comprehensive state-level panel of annual income inequality measures (the panel may be obtained online at www.shsu.edu/eco_mwf/inequality.html). Although IRS income data has several important limitations, including the censoring of individuals below a threshold level of income, it has the unique feature of being 
available annually for each state since the year 1916. Current empirical research on income inequality has benefited primarily from the construction of two prior income inequality data sets: the international panel of Deininger and Squire (1996), and the U.S time-series data of Piketty and Saez (2003). Deininger and Squire (1996) offer inequality measures for a wide panel of nations with several time-series observations for each nation beginning in the year 1960. These time-series observations are spaced over multiple decades, with an average of six observations per nation in their high-quality subset of the panel. ${ }^{2}$ Piketty and Saez (2003), on the other hand, construct a high-frequency U.S. time-series data set. Unlike the large- $N$ small- $T$ panel of Deininger and Squire, the Piketty and Saez data contains up to 85 annual observations for the U.S. covering the period 1913-1998.

This paper seeks to contribute to the literature on income inequality by providing a third data alternative: a panel which covers an under-exploited unit of observation, U.S. states, and that is large in both cross-sections and time-series observations. While a panel of U.S. states is more homogenous than most cross-national panels, it still retains a useful degree of heterogeneity derived from each state's unique political/institutional history, and regional heritage. Moreover, a moderate amount of cross-sectional heterogeneity would appear to be a useful econometric feature, as the overwhelming cross-sectional heterogeneity in the international panel of Deininger and Squire (see Li, Squire, and Zou, 1998) has led to some econometric misuse, a point of emphasis in Quah (2001) and Partridge (2005).

Our new state-level panel shows that many states followed a distinctive U-shaped pattern over the past century, with inequality peaking both before the Great Depression and again at the time of the new millennium. This trend is consistent with overall U.S. trends (see Piketty and Saez, 2003), though we do uncover sizable state-level variability over time. This variability is particularly large before the year 1945, and appears to reemerge during recent years. We also find considerable regional variation, with Northeastern states being most closely associated with aggregate U.S. trends, and Western states being the least associated.

A distinctive feature of our data is its unusually large panel dimensions $(N=$ $51, T=90)$. As econometric attention has recently shifted towards the asymptotics of large- $N$ large- $T$ macro panels (see for example, Pesaran and Smith, 1995; Pesaran, Shin and Smith, 1999; Phillips and Moon, 1999, 2000), the dimensions of our panel are large enough to exploit these developments. Alternatively, given the large number of time-series observations available for each state, traditional time-series 
analysis could be performed on each state individually, as one would with the U.S. time-series data of Piketty and Saez (2003). Likewise, the number of cross-sections is large enough for one to sub-sample the time-series observations (e.g. at five, ten, or twenty year intervals) and pursue the use of traditional large- $N$ small- $T$ panel data econometrics, as one would with the international panel of Deininger and Squire (1996).

The structure of the paper is as follows. Section 2 introduces our new panel and offers a brief overview of the trends in state-level income inequality. Section 3 continues this overview by presenting a comparison of the state-level inequality trends to aggregate U.S. inequality trends. Section 4 provides an important discussion of the key limitations inherent with IRS income data. Section 5 presents several alternative measures of income inequality, and compares these with the top income share measures presented in Sections 3 and 4. Finally, Section 6 offers a brief set of conclusions.

\section{TRENDS IN STATE-LEVEL INCOME INEQUALITY}

This paper provides a new panel annual state-level income inequality measures. The panel includes the 50 states and the District of Columbia, with 90 annual observations for each state except Alaska. For Alaska, the panel includes annual observations only for the period of statehood (1959 - 2005). This brings the total number of observations to 4,547 .

The inequality measures are constructed using data published in the IRS's Statistics of Income on the number of returns and adjusted gross income (before taxes) by state and by size of the adjusted gross income. ${ }^{3}$ The pre-tax adjusted gross income reported by the IRS is a broad measure of income. In addition to wages and salaries, it also includes capital income (dividends, interest, rents, and royalties) and entrepreneurial income (self-employment, small businesses, and partnerships). ${ }^{4}$ Notable income exclusions include interest on state and local bonds, and transfer income from federal and state governments. Further details on the construction of the inequality measures are provided in the Appendix. 


\section{Table 1}

\section{Descriptive Statistics of the Top Income Shares}

\begin{tabular}{|c|c|c|c|c|c|c|}
\hline \multirow[b]{2}{*}{ Variable } & \multirow[b]{2}{*}{ Mean } & \multirow[b]{2}{*}{$\begin{array}{l}\text { Standard } \\
\text { Deviation }\end{array}$} & \multirow{2}{*}{$\begin{array}{l}\text { Min. } \\
\text { Annual } \\
\text { Mean } \\
\text { (Year) }\end{array}$} & \multirow{2}{*}{$\begin{array}{c}\text { Max. } \\
\text { Annual } \\
\text { Mean } \\
\text { (Year) }\end{array}$} & \multicolumn{2}{|c|}{$\begin{array}{c}\text { Variance } \\
\text { Decomposition }\end{array}$} \\
\hline & & & & & $\begin{array}{c}\text { Across } \\
\text { State }\end{array}$ & $\begin{array}{l}\text { Over } \\
\text { Time }\end{array}$ \\
\hline $\begin{array}{l}\text { Top 1\% } \\
\text { Income } \\
\text { Share }\end{array}$ & $11.3 \%$ & 4.3 & 7.5 (1978) & 20.3 (2005) & $27.8 \%$ & $72.0 \%$ \\
\hline $\begin{array}{l}\text { Top 10\% } \\
\text { Income } \\
\text { Share }\end{array}$ & $34.5 \%$ & 5.9 & 28.2 (1953) & 45.5 (1916) & $23.0 \%$ & $77.1 \%$ \\
\hline
\end{tabular}

One must be cautious when using IRS income data, however, given the truncation of individuals at the low-end of the income distribution. For this reason, we will follow Piketty and Saez (2003) in focusing our attention on top income shares as primary indicators of inequality trends. Descriptive statistics for the top $1 \%$ and top $10 \%$ income shares are presented in Table 1 . Figure 1 presents the annual trends in these two income shares averaged over the states. Shaded areas show periods of recession as defined by the National Bureau of Economic Research (NBER). Both measures of inequality display a distinct U-shaped pattern over the sample period. In the early part of the century, the state-averaged top decile peaked at $45.5 \%$ in 1916 , and again at $44.1 \%$ in 1928 . Thereafter, the top decile began a substantial decline, particularly during the Great Depression and World War II (see also Goldin and Margo 1992). The income share of the top $10 \%$ fell to a sample-low of $28.2 \%$ in 1953 . After decades of post-World War II stability, large increases in inequality began in the 1980s, with a significant part of this increase occurring after the Tax Reform Act of 1986 (see also Levy and Murnane 1992, Gottschalk 1997, and Krueger 2003). By the final year of the sample, the top decile share reached $43.8 \%$, a level just below the 1916 peak, and the second highest value in the ninety year sample.

The state-averaged top $1 \%$ share of income followed a similar pattern. After early peaks in 1916 and 1928 (17.2\% and 17.8\%, respectively), the top $1 \%$ declined substantially in the 1930 s. Following a prolonged four-decade period of gradual decay, the top $1 \%$ attained a sample minimum of $7.5 \%$ in 1978 . Substantial increases followed in the $1980 \mathrm{~s}$, with the top $1 \%$ reaching a ninety-year sample-peak of $20.3 \%$ in 2005 . 
Figure 1

\section{Trends in Average State-Level Income Inequality}

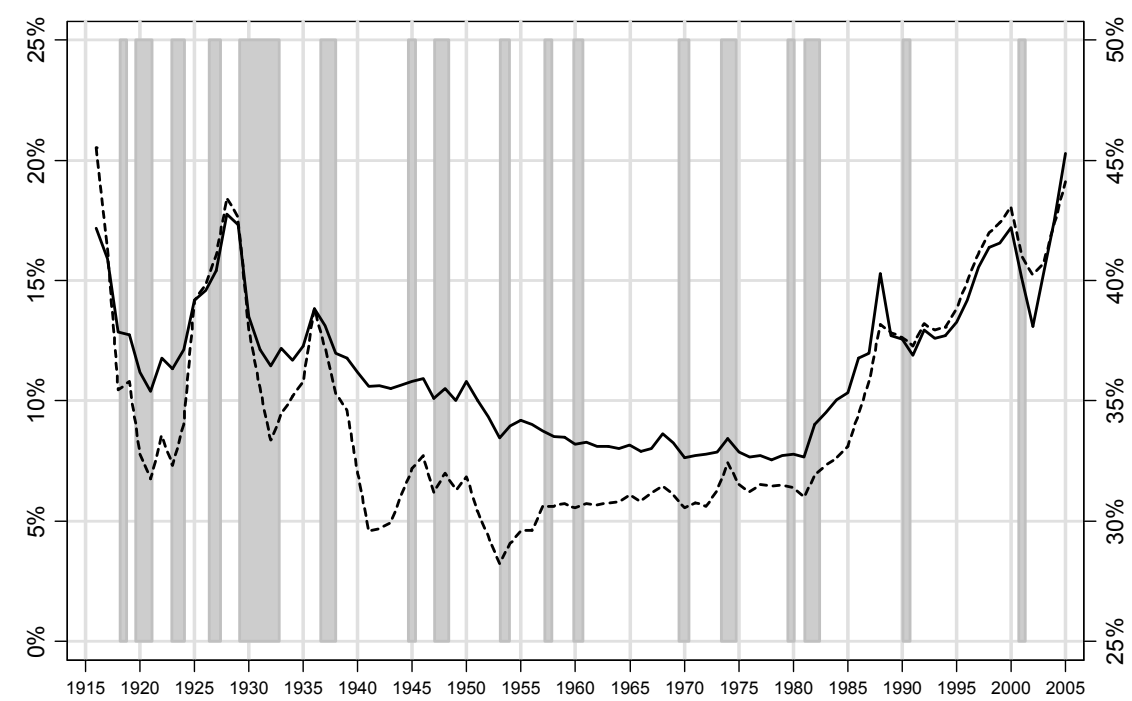

— Top 1\% (left scale) --------- Top 10\% (right scale) —_ NBER Recession

Table 1 also presents an analysis of variance for the two top income shares. Approximately three-fourths of the variation in inequality is due to variation through time, while one-quarter is due to variation across states. This decomposition contrasts with the international inequality panel of Deininger and Squire (1996), where approximately $90 \%$ of the variation is cross-sectional, while only $10 \%$ is through time (see Li, Squire, and Zou 1998, and Quah 2001). Two implications arise from these differences: first, the state-level panel is more balanced in its variation, and second, unlike the international panel, the state-level panel varies predominately through time, rather than across sections. The second feature is noteworthy given the econometric problems that arise with the common use of fixed-effect or first-difference estimators when a substantial proportion of the variation occurs through the cross-sections (see Barro 2000, Quah 2001, and Partridge 2005). 
Table 2

Top Decile Income Shares by State and Decade

\begin{tabular}{|c|c|c|c|c|c|c|c|c|c|c|c|c|c|c|c|c|c|c|c|c|c|c|c|c|}
\hline 赵告 & 穴 & $\begin{array}{l}\infty \\
\dot{\theta} \\
\dot{\theta}\end{array}$ & $\begin{array}{l}\hat{\infty} \\
\infty \\
\infty\end{array}$ & $\begin{array}{l}\stackrel{\sim}{\sim} \\
\stackrel{y}{f}\end{array}$ & 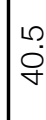 & 今 & $\begin{array}{l}10 \\
\mathscr{P} \\
\forall\end{array}$ & $\begin{array}{l}0 \\
0 \\
0 \\
10\end{array}$ & $\frac{\rho}{\dot{\sigma}}$ & $\left|\begin{array}{l}0 \\
\dot{L} \\
\end{array}\right|$ & 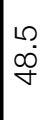 & 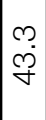 & \begin{tabular}{|l|}
$\infty$ \\
$\stackrel{m}{ }$ \\
\end{tabular} & $\begin{array}{l}0 \\
\dot{P} \\
\dot{\gamma}\end{array}$ & $\begin{array}{l}0 \\
\dot{f}\end{array}$ & $\bar{\infty}$ & $\begin{array}{l}\Omega \\
0 \\
\dot{0}\end{array} \mid$ & $\begin{array}{l}\infty \\
\dot{m} \\
\end{array}$ & $\begin{array}{l}\bar{\rho} \\
\dot{m}\end{array}$ & $\frac{\dot{\sigma}}{\dot{\sigma}}$ & \begin{tabular}{|l|}
$\infty$ \\
$\infty$ \\
$\infty$ \\
$\infty$
\end{tabular} & $\frac{\gamma}{\tilde{\gamma}}$ & 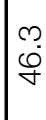 & ১. \\
\hline ڤ్ & $\begin{array}{l}m \\
\text { p. }\end{array}$ & $\begin{array}{l}\hat{N} \\
\infty \\
\infty\end{array}$ & $\begin{array}{l}0 \\
\stackrel{+}{O}\end{array}$ & गे & $\begin{array}{l}m \\
\infty \\
\infty\end{array}$ & $\begin{array}{l}0 \\
\mathscr{\gamma}\end{array}$ & 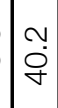 & $\mid \begin{array}{l}\checkmark \\
\dot{\sigma}\end{array}$ & $\begin{array}{l}\text { の } \\
\hat{m}\end{array}$ & $\left|\begin{array}{l|}\infty \\
\dot{\nu} \\
\dot{\gamma}\end{array}\right|$ & $\begin{array}{l}\stackrel{8}{8} \\
\dot{J}\end{array}$ & 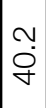 & $\begin{array}{l}\tilde{\sim} \\
\hat{m}\end{array}$ & $\begin{array}{l}\bar{\sigma} \\
\infty \\
\infty\end{array}$ & \begin{tabular}{|c|}
0 \\
$\dot{\sigma}$ \\
$\dot{\gamma}$
\end{tabular} & $\begin{array}{l}\hat{0} \\
\dot{e}\end{array}$ & $\left|\begin{array}{l}\Omega \\
\dot{\rho} \\
\rho\end{array}\right|$ & $\begin{array}{l}0 \\
\infty \\
\infty\end{array}$ & $\begin{array}{l}\hat{i} \\
\hat{m}\end{array}$ & 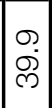 & \begin{tabular}{|l|}
0 \\
0 \\
0 \\
$e$
\end{tabular} & $\begin{array}{l}\sim \\
\infty \\
\infty \\
\infty\end{array}$ & 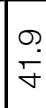 & $\hat{\hat{N}}$ \\
\hline$\stackrel{\infty}{\circ}$ & $\begin{array}{l}\text { क् } \\
\text { ले }\end{array}$ & $\begin{array}{l}\sim \\
\tilde{m} \\
m\end{array}$ & लि & $\begin{array}{l}N \\
\text { J }\end{array}$ & $\begin{array}{l}0 \\
\tilde{m} \\
\tilde{m}\end{array}$ & $\begin{array}{l}0 \\
0 \\
ల\end{array}$ & 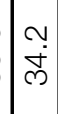 & $\begin{array}{l}\dot{ } \\
\dot{0} \\
\text { ले }\end{array}$ & $\begin{array}{l}\infty \\
\ddot{n} \\
m\end{array}$ & $\begin{array}{c}\tau \\
\dot{O} \\
\end{array}$ & $\begin{array}{l}10 \\
\infty \\
\infty \\
\infty\end{array}$ & $\begin{array}{l}0 \\
\dot{m}\end{array}$ & \begin{tabular}{|l|}
0 \\
$\tilde{m}$ \\
$m$
\end{tabular} & $\begin{array}{l}0 \\
\tilde{j} \\
\tilde{c}\end{array}$ & $\begin{array}{l}\omega \\
\stackrel{0}{0} \\
\tilde{m}\end{array}$ & $\frac{\bar{c}}{\bar{m}}$ & $\begin{array}{l}\simeq \\
\approx \\
ల\end{array} \mid$ & $\begin{array}{l}0 \\
\dot{\varpi}\end{array}$ & \begin{tabular}{|l|}
$\infty$ \\
$\tilde{m}$ \\
$m$
\end{tabular} & \begin{tabular}{|l|}
$m$ \\
$\omega$ \\
$\infty$ \\
$\infty$
\end{tabular} & $\frac{n}{m}$ & $\begin{array}{l}\cong \\
\tilde{n} \\
\tilde{n}\end{array}$ & 户े & ָ̃ \\
\hline$\frac{\mathscr{2}}{2}$ & 吕 & $\frac{N}{m}$ & $\begin{array}{l}\infty \\
\infty \\
\infty\end{array}$ & స. & ๗̃ & $\frac{\infty}{\infty}$ & $\frac{\dot{\sigma}}{\dot{m}}$ & $\begin{array}{l}0 \\
\tilde{N} \\
\tilde{\sigma}\end{array}$ & $\begin{array}{l}\sim \\
\mathbb{N} \\
\tilde{N}\end{array}$ & $\mid \begin{array}{l}\overline{0} \\
\dot{e}\end{array}$ & $\begin{array}{l}\sim \\
\stackrel{y}{ }\end{array}$ & $\begin{array}{l}\circ \\
\stackrel{n}{n} \\
m\end{array}$ & $\frac{N}{\infty}$ & $\frac{N}{\infty}$ & $\begin{array}{l}\dot{v} \\
\dot{p}\end{array}$ & $\begin{array}{l}\text { m. } \\
\stackrel{\sim}{N}\end{array}$ & $\begin{array}{l}m \\
0 \\
0 \\
0\end{array}$ & $\begin{array}{l}0 \\
\dot{m}\end{array}$ & $\frac{N}{\infty}$ & 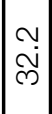 & \begin{tabular}{|l|}
$\infty$ \\
$\dot{\infty}$ \\
\end{tabular} & 苂. & & $\begin{array}{l}\simeq \\
\stackrel{N}{N}\end{array}$ \\
\hline 。̊̊ & ๑ & $\begin{array}{l}10 \\
0 \\
0\end{array}$ & $\hat{\curvearrowright}$ & $\hat{0}$ & $\begin{array}{l}0 \\
\sim \\
\tilde{m}\end{array}$ & $\hat{\circ}$ & 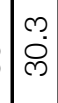 & $\begin{array}{l}\widetilde{N} \\
\widetilde{N} \\
\tilde{n}\end{array}$ & $\hat{\aleph}$ & $\begin{array}{l}0 \\
\stackrel{0}{0} \\
\tilde{\omega}\end{array}$ & $\hat{m}$ & $\begin{array}{l}0 \\
\text { ஸे } \\
\text { లె }\end{array}$ & $\frac{\dot{\sigma}}{\dot{\sigma}}$ & $\begin{array}{l}0 \\
\stackrel{\mathfrak{S}}{\mathbf{N}}\end{array}$ & $\begin{array}{l}0 \\
0 \\
8 \\
0\end{array}$ & $\underset{\sim}{\stackrel{D}{\sim}}$ & $\begin{array}{l}\text { の } \\
\stackrel{\text { N }}{ }\end{array}$ & $\begin{array}{l}0 \\
\dot{\infty}\end{array}$ & 高 & \begin{tabular}{|l|} 
\\
\\
\end{tabular} & $\begin{array}{l}0 \\
\stackrel{2}{N}\end{array}$ & ¿̊. & ๓n & Г্ণ \\
\hline ஜू & 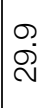 & $\begin{array}{l}\infty \\
\dot{N} \\
\infty\end{array}$ & ' & $\begin{array}{l}0 \\
0 \\
0\end{array}$ & 吕 & $\begin{array}{l}\infty \\
\stackrel{N}{N}\end{array}$ & $\begin{array}{l}\forall \\
\dot{\rho} \\
\end{array}$ & $\frac{0}{0}$ & $\begin{array}{l}\infty \\
\dot{\infty} \\
\dot{m}\end{array}$ & $\left|\begin{array}{l}m \\
\tilde{N} \\
\tilde{c}\end{array}\right|$ & $\begin{array}{l}\sim \\
\tilde{m} \\
\tilde{m}\end{array}$ & 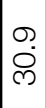 & $\begin{array}{l}\forall \\
\dot{N} \\
\stackrel{N}{*}\end{array}$ & $\begin{array}{l}0 \\
\infty \\
\sim \\
\sim\end{array}$ & 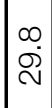 & $\begin{array}{l}0 \\
\text { N } \\
\text { a }\end{array}$ & $\overline{\stackrel{N}{N}}$ & $\begin{array}{l}0 \\
\stackrel{2}{N}\end{array}$ & $\begin{array}{l}\check{8} \\
\dot{0}\end{array}$ & $\frac{\sim}{\infty}$ & $\begin{array}{l}\dot{v} \\
\dot{N} \\
\text { N }\end{array}$ & হं & ब্ & $\begin{array}{l}\checkmark \\
\stackrel{\sim}{\sim} \\
\stackrel{N}{N}\end{array}$ \\
\hline 里 & $\frac{N}{m}$ & 每 & I & O & $\begin{array}{l}n \\
\tilde{m} \\
m\end{array}$ & ১. & $\begin{array}{l}\infty \\
\sim \\
\tilde{m}\end{array}$ & $\begin{array}{l}10 \\
\tilde{N} \\
\tilde{n}\end{array}$ & $\hat{\stackrel{N}{\mathcal{F}}}$ & $\begin{array}{l}\overline{\check{c}} \\
\tilde{c}\end{array}$ & $\begin{array}{l}10 \\
0 \\
0 \\
0\end{array}$ & $\hat{m}$ & $\begin{array}{l}N \\
0 \\
0 \\
p\end{array}$ & $\begin{array}{l}N \\
\stackrel{N}{N}\end{array}$ & 吕 & $\begin{array}{l}L \\
\infty \\
\infty \\
\sim\end{array}$ & $\begin{array}{l}n \\
0 \\
0\end{array}$ & $\frac{n}{\infty}$ & \begin{tabular}{|l|}
0 \\
0 \\
$\infty$
\end{tabular} & $\frac{n}{\bar{m}}$ & $\begin{array}{l}N \\
0 \\
0 \\
e\end{array}$ & $\frac{n}{m}$ & $\frac{\nabla}{\dot{m}}$ & $\begin{array}{l}\stackrel{0}{0} \\
\stackrel{\sim}{\sim}\end{array}$ \\
\hline ஜ̊ & $\begin{array}{l}\infty \\
\omega \\
\infty\end{array}$ & $\begin{array}{l}\qquad 0 \\
\ddot{n} \\
\ddot{n}\end{array}$ & ' & $\begin{array}{l}\stackrel{N}{N} \\
\stackrel{N}{N}\end{array}$ & 离 & $\begin{array}{l}0 \\
0 \\
0\end{array}$ & $\begin{array}{l}\dot{v} \\
\tilde{m}\end{array}$ & $\begin{array}{l}0 \\
\dot{\gamma} \\
\stackrel{\gamma}{*}\end{array}$ & $\frac{\varphi}{\bar{\sigma}}$ & $\left|\begin{array}{l}0 \\
\tilde{m} \\
\tilde{m}\end{array}\right|$ & $\begin{array}{l}\stackrel{0}{\mathfrak{j}} \\
\underset{f}{2}\end{array}$ & $\begin{array}{l}\sim \\
\stackrel{n}{\infty} \\
\infty\end{array}$ & 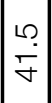 & $\begin{array}{l}\infty \\
\stackrel{\infty}{\sim} \\
\stackrel{\sim}{\sim}\end{array}$ & $\begin{array}{l}0 \\
\dot{\rho} \\
\tilde{m}\end{array}$ & $\bar{c}$ & $\begin{array}{l}\hat{\sim} \\
\tilde{c}\end{array}$ & $\begin{array}{l}\text { ๙ } \\
\text { } \\
\text { m. }\end{array}$ & \begin{tabular}{|l|}
$\hat{n}$ \\
$\rho$ \\
$\infty$
\end{tabular} & $\overline{\ddot{m}}$ & $\begin{array}{l}0 \\
\dot{\sigma} \\
\end{array}$ & مि. & ஜ & $\begin{array}{l}n \\
\infty \\
\infty\end{array}$ \\
\hline$\stackrel{\mathscr{\Omega}}{\sigma}$ & $\stackrel{r}{\tilde{m}}$ & $\begin{array}{l}0 \\
\dot{1} \\
\tilde{\infty}\end{array}$ & ' & $\begin{array}{l}0 \\
\text { ○े }\end{array}$ & $\begin{array}{l}n \\
\tilde{m} \\
\tilde{m}\end{array}$ & O̊. & \begin{tabular}{|l|}
$\infty$ \\
0 \\
$\infty$ \\
$\tilde{n}$
\end{tabular} & $\underset{⿱}{\stackrel{v}{*}}$ & $\begin{array}{l}\infty \\
\tilde{N} \\
\tilde{N}\end{array}$ & ळ & $\frac{\text { ra }}{\dot{\gamma}}$ & ¿. & $\stackrel{-}{\dot{P}}$ & $\begin{array}{l}0 \\
\stackrel{\leftrightarrow}{N} \\
\text { N }\end{array}$ & $\begin{array}{l}0 \\
\stackrel{O}{Y}\end{array}$ & $\begin{array}{l}0 \\
\stackrel{\circ}{\infty}\end{array}$ & $\begin{array}{l}0 \\
\tilde{m} \\
\tilde{m}\end{array}$ & $\begin{array}{l}m \\
\stackrel{n}{m}\end{array}$ & \begin{tabular}{|c|}
0 \\
$\infty$ \\
$\infty$ \\
$\infty$
\end{tabular} & 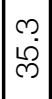 & $\begin{array}{l} \\
0 \\
0 \\
\end{array}$ & $\underset{\sim}{\stackrel{N}{\sim}}$ & & $\stackrel{\sim}{\stackrel{\sim}{\gamma}}$ \\
\hline 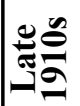 & 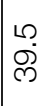 & $\begin{array}{l}\stackrel{0}{0} \\
\stackrel{0}{0}\end{array}$ & & $\begin{array}{l}0 \\
\ddot{\omega} \\
\mathscr{e}\end{array}$ & $\begin{array}{l}\text { } \\
\stackrel{\sim}{m}\end{array}$ & $\hat{O}$ & $\begin{array}{l}\overline{0} \\
\infty \\
\infty\end{array}$ & $\mid \begin{array}{l}\dot{\sigma} \\
\dot{\sigma}\end{array}$ & 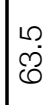 & $\begin{array}{l}\hat{\sim} \\
\stackrel{\vartheta}{*}\end{array}$ & Nִ & $\begin{array}{l}m \\
0 \\
0\end{array}$ & \begin{tabular}{|l|} 
\\
0 \\
0 \\
10
\end{tabular} & $\begin{array}{l}\vec{v} \\
\stackrel{\sim}{m}\end{array}$ & $\underset{f}{\check{f}}$ & $\begin{array}{l}\circ \\
\dot{0} \\
\rho\end{array}$ & $\begin{array}{l}\simeq \\
\stackrel{N}{ }\end{array}$ & $\begin{array}{l}m \\
\infty \\
m\end{array}$ & $\begin{array}{l}r \\
\tilde{n}\end{array}$ & $\begin{array}{l}0 \\
\dot{p} \\
\dot{q}\end{array}$ & ब़् & ஓं & $\begin{array}{l}\infty \\
\dot{\gamma}\end{array}$ & ஜ) \\
\hline$\approx$ & $\begin{array}{l}10 \\
\text { हैं }\end{array}$ & $\begin{array}{l}0 \\
\ddot{m} \\
\ddot{m}\end{array}$ & $\begin{array}{l}\widetilde{N} \\
\tilde{m}\end{array}$ & $\begin{array}{l}\infty \\
\sim \\
\sim \\
\text { ஸे }\end{array}$ & $\begin{array}{l}\text { Ð } \\
\tilde{m}\end{array}$ & $\begin{array}{l}\circ \\
0 \\
0\end{array}$ & $\begin{array}{l}0 \\
\stackrel{0}{0} \\
\infty\end{array}$ & $\begin{array}{l}10 \\
0 \\
0 \\
0\end{array}$ & $\begin{array}{l}\stackrel{\sim}{~} \\
\stackrel{P}{+}\end{array}$ & Г্ & $\begin{array}{l}\hat{\infty} \\
\infty \\
\infty\end{array}$ & $\begin{array}{l}0 \\
\stackrel{0}{ } \\
\infty\end{array}$ & $\begin{array}{l}\hat{n} \\
\dot{\rho} \\
\infty\end{array}$ & \begin{tabular}{|l|}
0 \\
0 \\
0 \\
$\varnothing$
\end{tabular} & $\begin{array}{l}\sim \\
\stackrel{N}{0} \\
\tilde{e}\end{array}$ & 衣 & $\begin{array}{l}\circ \\
\text { ஸे } \\
\text { ले }\end{array}$ & ஸ் & $\begin{array}{l}\mathscr{} \\
\ddot{m} \\
\infty\end{array}$ & $\begin{array}{l}\hat{D} \\
\dot{j} \\
\text { ले }\end{array}$ & $\begin{array}{l}\stackrel{N}{*} \\
\dot{J}\end{array}$ & $\begin{array}{l}0 \\
\stackrel{0}{0} \\
\text { ले }\end{array}$ & त् & ले \\
\hline & 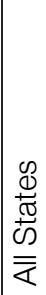 & $\begin{array}{l}\frac{\sigma}{\varepsilon} \\
\frac{\sigma}{\sigma} \\
\frac{O}{\sigma} \\
\frac{\sigma}{\mathbb{Z}}\end{array}$ & $\begin{array}{l}\frac{\pi}{v} \\
\frac{v}{0} \\
\frac{\pi}{\alpha} \\
\end{array}$ & $\begin{array}{l}\frac{\sigma}{c} \\
\frac{\mathrm{D}}{N} \\
\frac{\mathrm{L}}{\mathrm{L}}\end{array}$ & 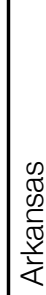 & 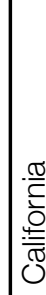 & $\begin{array}{l}0 \\
\frac{0}{0} \\
\frac{\pi}{0} \\
\text { OO } \\
\mathcal{O}\end{array}$ & 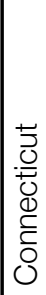 & $\mid \begin{array}{l}\frac{0}{0} \\
\frac{1}{3} \\
\frac{0}{0} \\
0 \\
0\end{array}$ & 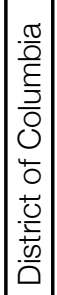 & $\begin{array}{l}\frac{\pi}{0} \\
\frac{0}{0} \\
\frac{0}{4}\end{array}$ & \begin{tabular}{|l}
$\frac{\sigma}{\sigma}$ \\
$\frac{0}{0}$ \\
$\mathbb{d}$ \\
0
\end{tabular} & 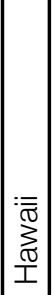 & $\left|\begin{array}{l}\frac{0}{0} \\
\frac{\pi}{0} \\
\frac{0}{0}\end{array}\right|$ & 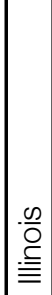 & 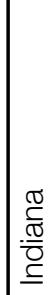 & 疋 & 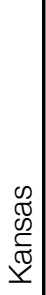 & 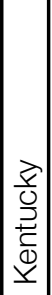 & 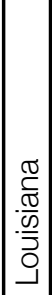 & 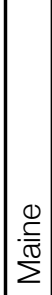 & 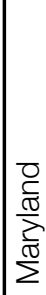 & 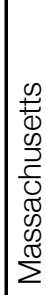 & 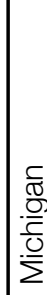 \\
\hline
\end{tabular}




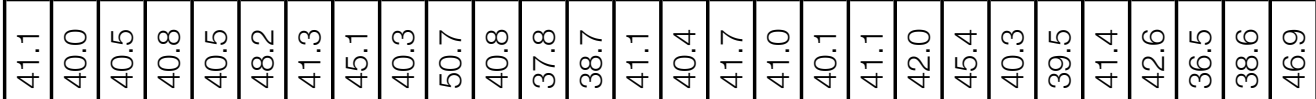

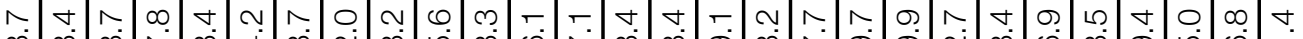

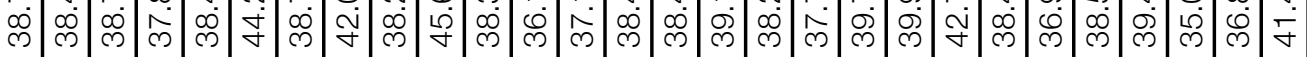

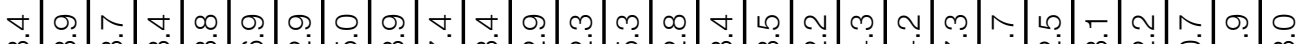
लூ లై

$の$ の ळ ले ल ल ल

N ரூ

几 হं m

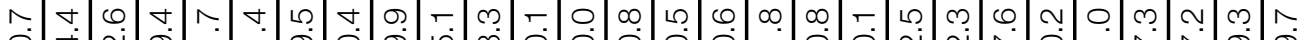

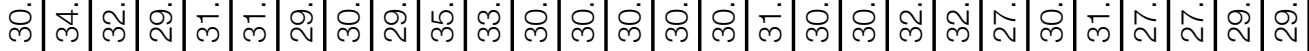

ల ஸे లై

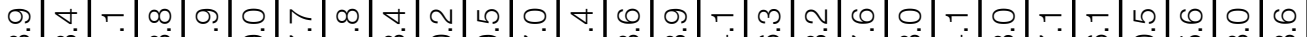
ळ

几 fं

0 ○

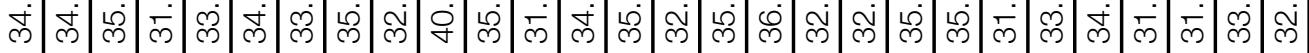

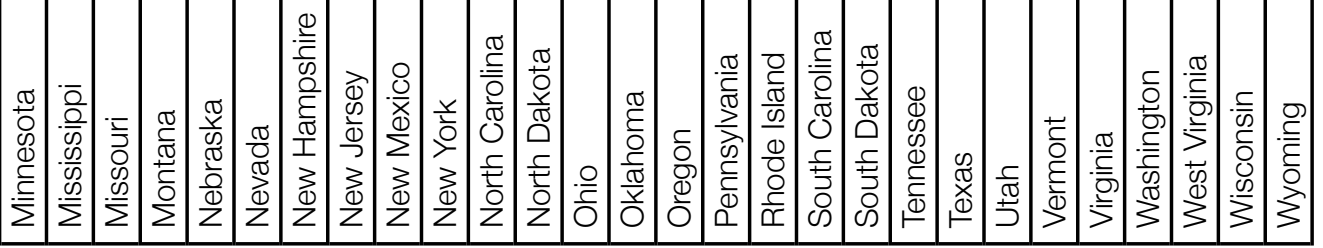


The distinguishing feature of our panel is the construction of annual inequality measures for each of the states. Table 2 shows the income shares of the top decile for each state averaged over the decades. Figure 2 shows the individual state-level trends in the top $1 \%$ and $10 \%$ income shares. ${ }^{5}$ The lowest level of income inequality over the ninety year period occurred in North Dakota (with an average top decile share of $30.6 \%$ ), while the highest level occurred in Delaware and New York (43.2\% and $40.6 \%$, respectively). Table 2 shows that the highest level of inequality over the sample period occurred most frequently in the early 2000s (33 of the states), or in the late 1910s (17 of the states). For a majority of the states, the minimum level of inequality occurred during the 1950s (33 states).

Comparing the state-level trends in the top decile presented in Figure 2 with the average-state trend presented in Figure 1, the average Pearson's correlation is 0.85. The four lowest Pearson's correlations occurred in North Dakota, South Dakota, Delaware, and Idaho $(0.39,0.48,0.51$, and 0.57 respectively). The remaining states vary between a correlation of 0.67 and 0.99 (Virginia and New Mexico, respectively), with an average correlation of 0.89 . Twenty-three of the states in fact have a very strong correlation (over 0.90 ) with the average-state.

Perhaps the most salient feature of income inequality trends over the past century is the distinct $U$-shaped pattern noted in Figure 1. To further evaluate this pattern, in Table 3 we estimate the quadratic equation Top $10 \%=\beta_{0}+\beta_{1}$ year $+\beta_{2}$ year $^{2}$ for the average-state and each individual state. From this estimation, $\beta_{2}>0$ indicates a U-shaped parabola, while $\beta_{2}<0$ indicates an upside down U-shaped parabola. In all cases except Alaska, $\beta_{2}$ is both positive and statistically significant at the $1 \%$ level, indicating a $U$-shaped function that opens upward. Moreover, we can estimate the minimum year of the parabola as $-\beta_{1} /\left(2 \beta_{2}\right)$. Most states have this estimated vertex in the 1940s, 1950s, or 1960s (45 of the states plus D.C.). The remaining five states, however, are outliers with an estimated minimum that is either before this period (Alaska, North Dakota, South Dakota, and Idaho), or after (Delaware). Hence, with the possible exception of these five states, the fit of a U-shaped pattern appears reasonable and consistent.

\section{COMPARISON OF STATE-LEVEL INEQUALITY TO U.S. INEQUALITY}

Aggregate U.S. trends in income inequality from IRS income data have been explored before, most notably by Piketty and Saez (2003), who construct several 
Figure 2

Individual State-Level Trends in Income Inequality

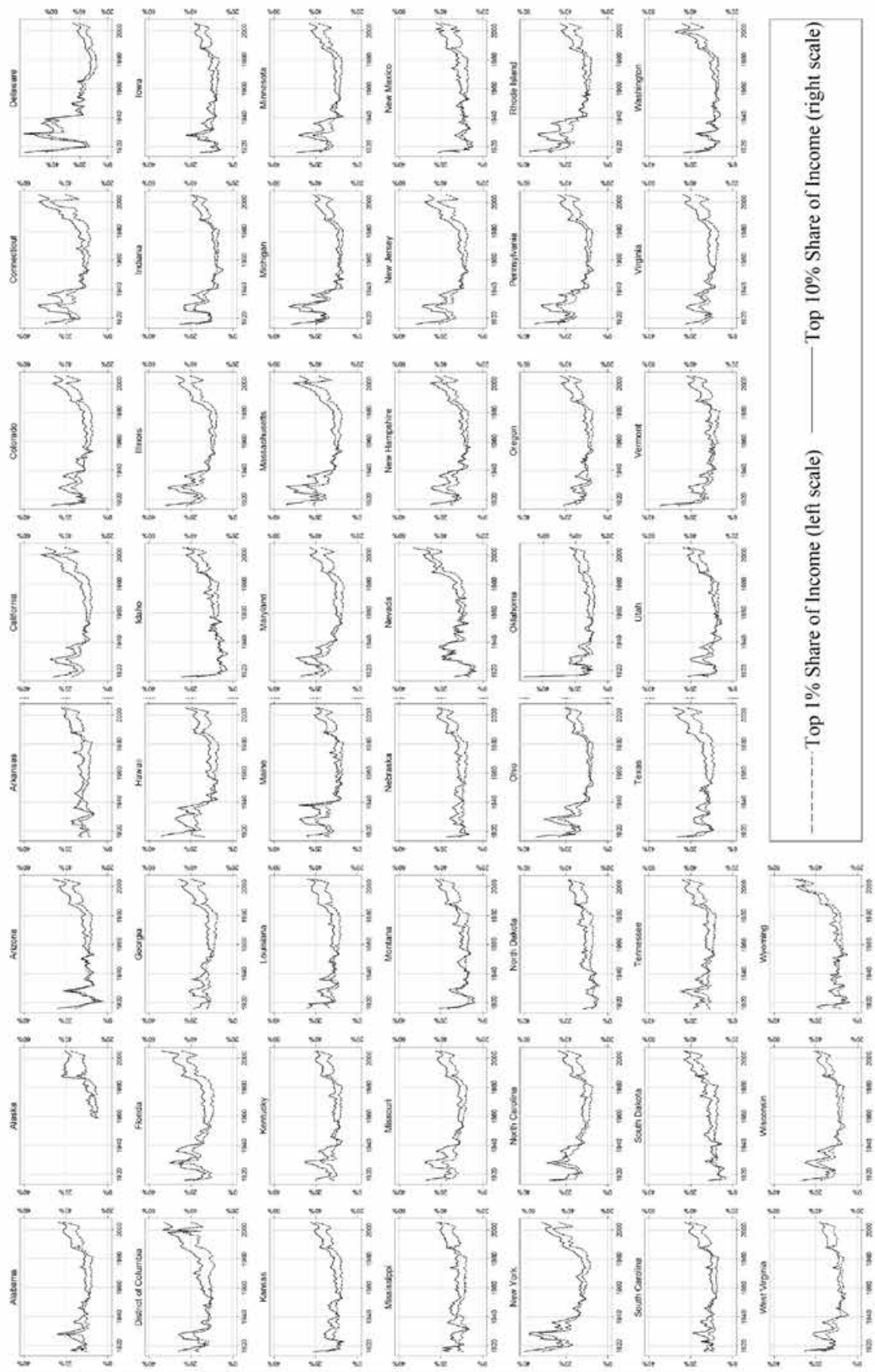




\section{Table 3}

\section{Top Decile Income Shares by State and Decade}

\begin{tabular}{|l|c|c|c|c|c|}
\hline & $\boldsymbol{\beta 0}$ & $\boldsymbol{\beta 1}$ & $\mathbf{( \mathbf { \beta 2 }}$ & $\mathbf{R 2}$ & Minimum \\
\hline All States & 226.3 & -0.231 & 0.589 & 69.3 & 1958.1 \\
\hline Alabama & 182.0 & -0.186 & 0.475 & 65.7 & 1955.6 \\
\hline Alaska & $28.1^{\star}$ & $-0.032^{*}$ & $0.089^{\star}$ & 77.7 & $1778.1^{*}$ \\
\hline Arizona & 172.8 & -0.177 & 0.455 & 67.4 & 1947.8 \\
\hline Arkansas & 96.6 & -0.099 & 0.254 & 58.6 & 1947.2 \\
\hline California & 326.3 & -0.333 & 0.851 & 79.2 & 1956.7 \\
\hline Colorado & 227.1 & -0.232 & 0.592 & 60.8 & 1957.5 \\
\hline Connecticut & 380.1 & -0.388 & 0.989 & 69.2 & 1959.6 \\
\hline Delaware & 262.2 & -0.264 & 0.667 & 51.3 & 1982.0 \\
\hline District of Columbia & 278.2 & -0.285 & 0.729 & 79.3 & 1952.3 \\
\hline Florida & 234.5 & -0.239 & 0.612 & 51.4 & 1956.2 \\
\hline Georgia & 185.2 & -0.189 & 0.484 & 68.0 & 1955.0 \\
\hline Hawaii & 310.3 & -0.315 & 0.802 & 74.9 & 1965.9 \\
\hline Idaho & 122.8 & -0.127 & 0.327 & 75.5 & 1936.4 \\
\hline Illinois & 334.9 & -0.341 & 0.870 & 72.7 & 1961.1 \\
\hline Indiana & 227.8 & -0.232 & 0.592 & 54.1 & 1960.3 \\
\hline lowa & 106.6 & -0.109 & 0.279 & 38.1 & 1952.7 \\
\hline Kansas & 137.6 & -0.141 & 0.361 & 63.1 & 1950.8 \\
\hline Kentucky & 190.3 & -0.194 & 0.495 & 58.7 & 1959.6 \\
\hline Louisiana & 206.2 & -0.211 & 0.538 & 74.4 & 1956.2 \\
\hline Maine & 232.9 & -0.237 & 0.603 & 53.5 & 1964.5 \\
\hline Maryland & 287.5 & -0.293 & 0.745 & 74.2 & 1963.0 \\
\hline Massachusetts & 393.6 & -0.401 & 1.021 & 78.0 & 1962.3 \\
\hline Michigan & 313.0 & -0.319 & 0.811 & 66.2 & 1963.8 \\
\hline Minnesota & 245.9 & -0.251 & 0.639 & 62.6 & 1960.5 \\
\hline Mississippi & 118.1 & -0.121 & 0.309 & 57.1 & 1952.3 \\
\hline Missouri & 240.8 & -0.245 & 0.624 & 66.6 & 1963.0 \\
\hline Montana & 132.8 & -0.136 & 0.351 & 70.4 & 1943.4 \\
\hline Nebraska & 108.5 & -0.111 & 0.286 & 66.5 & 1946.2 \\
\hline Nevada & 166.1 & -0.171 & 0.440 & 62.7 & 1941.2 \\
\hline New Hampshire & 269.2 & -0.274 & 0.700 & 66.2 & 1960.1 \\
\hline New Jersey & 347.2 & -0.354 & 0.903 & 77.0 & 1959.9 \\
\hline New Mexico & 112.4 & -0.116 & 0.298 & 70.5 & 1940.6 \\
\hline
\end{tabular}




\begin{tabular}{|l|c|c|c|c|c|}
\hline New York & 447.1 & -0.455 & 1.159 & 79.3 & 1962.9 \\
\hline North Carolina & 220.4 & -0.224 & 0.571 & 59.5 & 1963.3 \\
\hline North Dakota & 27.2 & -0.029 & 0.076 & 78.9 & 1879.5 \\
\hline Ohio & 299.3 & -0.305 & 0.775 & 68.5 & 1964.1 \\
\hline Oklahoma & 235.0 & -0.239 & 0.611 & 43.5 & 1960.1 \\
\hline Oregon & 206.8 & -0.211 & 0.540 & 77.8 & 1955.7 \\
\hline Pennsylvania & 351.7 & -0.358 & 0.911 & 74.5 & 1963.8 \\
\hline Rhode Island & 359.0 & -0.365 & 0.928 & 79.6 & 1966.4 \\
\hline South Carolina & 159.1 & -0.163 & 0.416 & 69.9 & 1953.2 \\
\hline South Dakota & 65.5 & -0.068 & 0.178 & 81.9 & 1916.6 \\
\hline Tennessee & 197.8 & -0.202 & 0.516 & 67.7 & 1957.2 \\
\hline Texas & 199.8 & -0.204 & 0.524 & 75.8 & 1951.0 \\
\hline Utah & 220.1 & -0.225 & 0.575 & 65.9 & 1954.8 \\
\hline Vermont & 238.4 & -0.243 & 0.619 & 68.7 & 1960.9 \\
\hline Virginia & 199.7 & -0.204 & 0.521 & 65.8 & 1957.4 \\
\hline Washington & 231.8 & -0.237 & 0.608 & 78.5 & 1951.5 \\
\hline West Virginia & 215.0 & -0.219 & 0.558 & 61.3 & 1960.9 \\
\hline Wisconsin & 244.8 & -0.249 & 0.636 & 65.3 & 1961.4 \\
\hline Wyoming & 227.3 & -0.233 & 0.598 & 77.3 & 1948.4 \\
\hline
\end{tabular}

Note: Estimated fit based on the quadratic equation: Top $10 \%=\beta_{0}+\beta_{1}$ year $+\beta_{2}$ year ${ }^{2}$, where $\beta_{2}>0$ indicates a U-shaped parabola, with the minimum year estimated by: $-\beta_{1} /\left(2 \beta_{2}\right)$.

*: Indicates estimated coefficients are not statistically significant at the $10 \%$ level. All other coefficients are statistically significant at the $1 \%$ level.

annual time-series measures of U.S. top income shares beginning in the year 1913 . Figures 3 presents a comparison of our new state-level inequality panel with the aggregate U.S. time-series data of Piketty and Saez. The solid line shows the trend in the (unweighted) state average of the top decile income share from our new state-level panel. The dashed line is the aggregate U.S. top decile income share from Piketty and Saez (2003). ${ }^{6}$ Individual points are the state-level observations from our new panel. 


\section{Figure 3}

\section{Comparison of Top $10 \%$ Income Share from the New State-Level Inequality Panel to the U.S. Time-Series Data of Piketty and Saez (2003)}

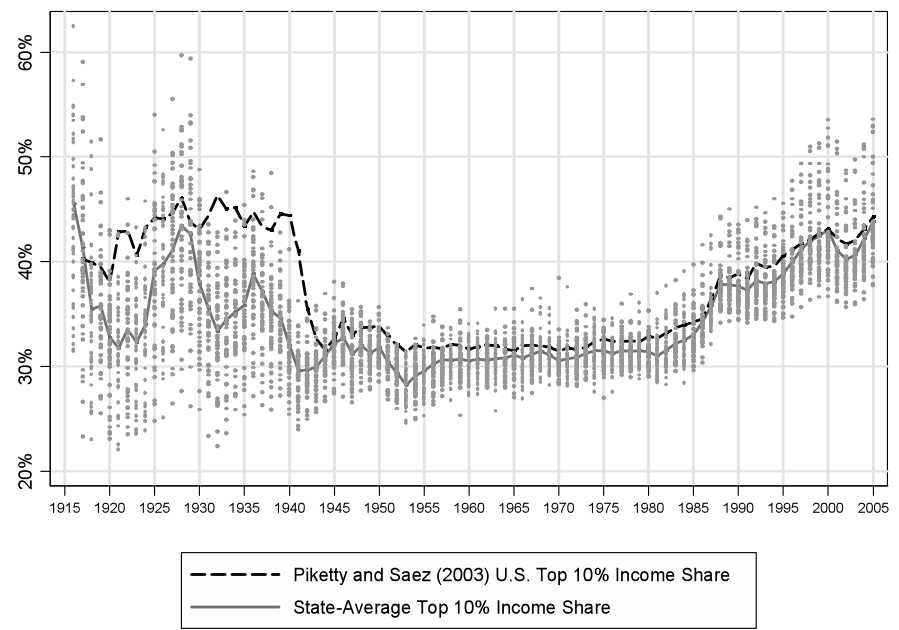

Though one would not expect an exact match, our unweighted state-average follows closely the aggregate U.S. inequality trend reported by Piketty and Saez, particularly after World War II. The mean top 10\% share of income averaged across the states from our panel is $34.5 \%$. In the U.S. time-series data of Piketty and Saez, the mean top decile share of income is $37.3 \%$ for the sample period. The minimum annual share of income is $28.2 \%$ in the state-level panel sample, and $31.4 \%$ in Piketty and Saez (both occurring in 1953), while the maximum annual share is $45.5 \%$ in our panel, and 46.3\% in Piketty and Saez (1916 and 1932, respectively). ${ }^{7}$ Overall, the Pearson's correlation coefficient between the two series is 0.77 . For the period after World War II, however, the Pearson's correlation increases to 0.99.

The tighter fit after 1945 is in part due to the greater degree of state-level variability before World War II. In the period 1916 to 1941, for example, the standard deviation of the top decile is 0.074 . After World War II the standard deviation decreases by about one-third, to 0.049 . It is noteworthy that the higher variability reemerges at the end of the sample period: if one considers only the five decade period 1945 to 1995 , the standard deviation of the top decile is only 0.034 , less than half the pre-World War II value.

Figure 4 provides a time-series comparison of each state's top decile to the 
U.S. top decile income share from the Piketty and Saez (2003) data. While the average Pearson's correlation among the states is 0.63 , there is notable regional variation. The Northeastern states most closely fit the overall U.S. trend, with correlation values above 0.83 for many of these states (Connecticut, Maine, Massachusetts, Maryland, New Hampshire, New York, Ohio, Pennsylvania, and Rhode Island). Many of the Western states, however, have a low correlation to the U.S. trend. Several in fact have correlation values of less than 0.10 (Arizona, Idaho, Montana, New Mexico, North Dakota, and South Dakota). ${ }^{8}$

\section{Figure 4}

\section{State-Level Correlations of the Top $10 \%$ Income Shares to the U.S. Time-Series Data of Piketty and Saez (2003)}

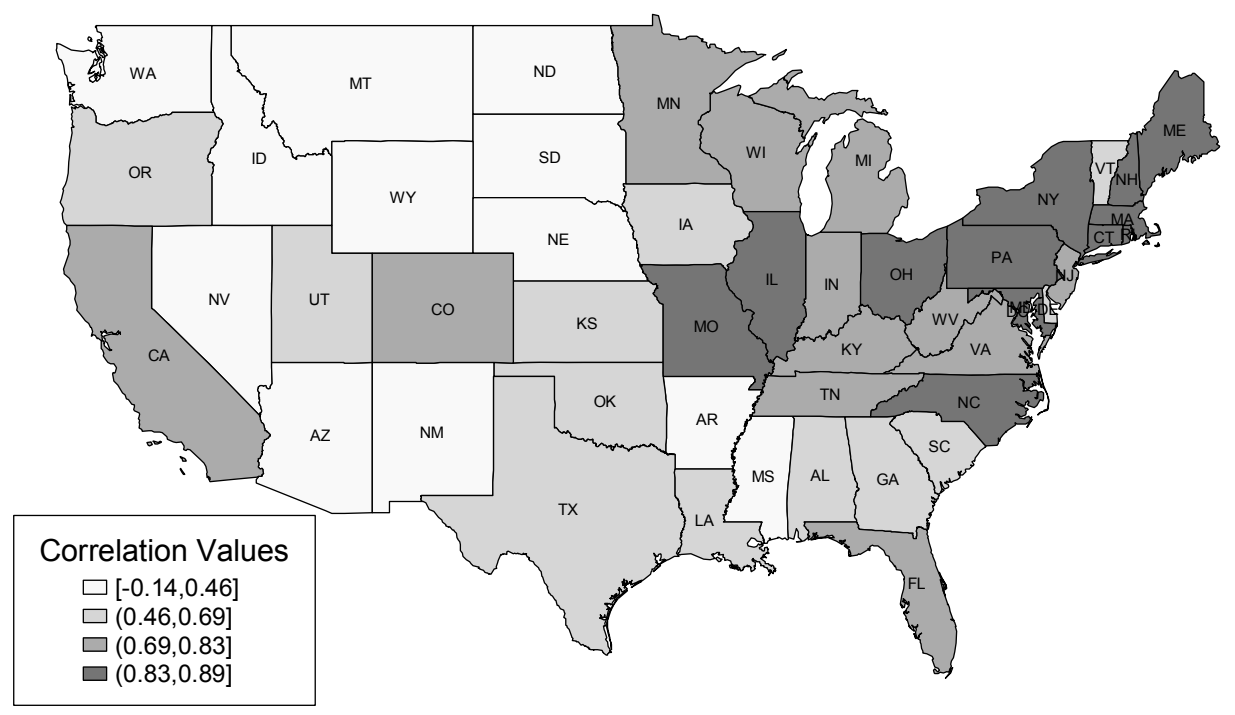

To the extent that our new panel will be used in future empirical research, it is noteworthy that the moderate state-level heterogeneity evident in Figure 4 offers several potentially important econometric advantages over the use of aggregate U.S. time-series data. The larger number of observations increases the degrees of freedom, and along with the enhanced variability, helps alleviate the multicollinearity problems which often plague time-series studies. It has been noted extensively elsewhere that panel data are also likely to improve the efficiency of the econometric estimates, reduce aggregation bias, and allow for the construction of more complicated econometric models (see for example, Baltagi 2005, p.4-9; Hsiao 2003, p.1-8). 


\section{LIMITATIONS OF IRS TAX DATA}

A significant limitation of IRS income data is the omission of some individuals earning less than a threshold level of gross income. This threshold varies by age and marital status, as well as the tax filing year. For this reason, we have followed Piketty and Saez (2003) in focusing on measures of top-income shares as our primary indicators of inequality. Other non-IRS data sources have the clear advantage of not omitting these low-income individuals, but these sources are either not available annually, such as the decennial Census, or, in the case of the March Current Population Survey (CPS), are only available annually for more recent years. Akhand and Liu (2002), moreover, provide evidence that these survey-based alternatives suffer additional bias resulting from an "over-reporting of earnings by individuals in the lower tail of the income distribution and under-reporting by individuals in the upper tail of the income distribution" (p. 258). The IRS, unlike the March CPS or Census Bureau, will penalize respondents for income reporting errors.

The omission of low-end income earners in IRS data is most problematic before the 1940s, when the number of tax returns filed each year was relatively small. Figure 5 displays the overall trends in the number of tax returns filed each year (see Hollenbeck and Kahr 2008), as well as trends in the size of the U.S. population. In the first few years after passage of the $16^{\text {th }}$ Amendment in 1913, the number of tax returns ranged from only 330 thousand to 440 thousand per year. As a result of significant tax law changes in 1916 and 1917, the number of tax returns rose to over 7.2 million in 1920. Over the following two decades, the number of returns grew little, climbing only to 7.7 million in 1939. The introduction of lower income filing requirements in 1940 caused the number of returns to increase rapidly in the early 1940s, surpassing 55 million in 1947. As Figure 5 shows, the yearly increases in the number of tax returns filed after 1947 follow closely the changes in the U.S. population.

\section{ADDITIONAL MEASURES OF INCOME INEQUALITY}

Figure 6 presents the annual trends in four additional measures of income inequality: the relative mean deviation, Gini coefficient, Atkinson index, and Theil's entropy index. The figure shows changes in each measure based on their 1916 values. Unlike the two top income shares, these four additional measures focus on assessing inequality over the entirety of the income distribution. While analytically more ap- 
pealing, this feature comes with a drawback in the context of IRS income data, given the truncation of some individuals at the low-end of the income distribution. Each of these four additional measures represents a different class of inequality measures (based on transfer principles and decomposability), with the relative mean deviation being the least analytically attractive, and the Theil entropy index being the most. ${ }^{9}$

\section{Figure 5}

\section{Comparison of the Number of Individual Income Tax Return Filed to the U.S. Population}

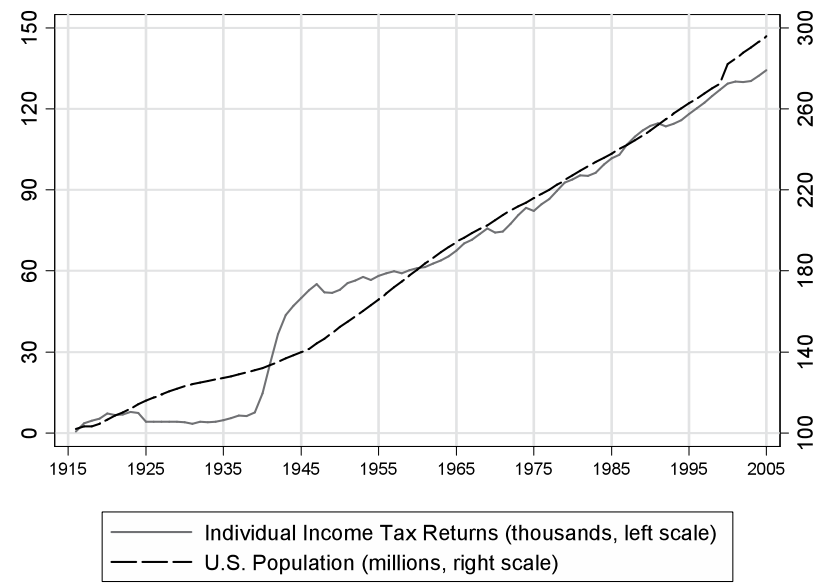

Figure 6

Comparison of Additional Income Inequality Measures $(1916=100)$

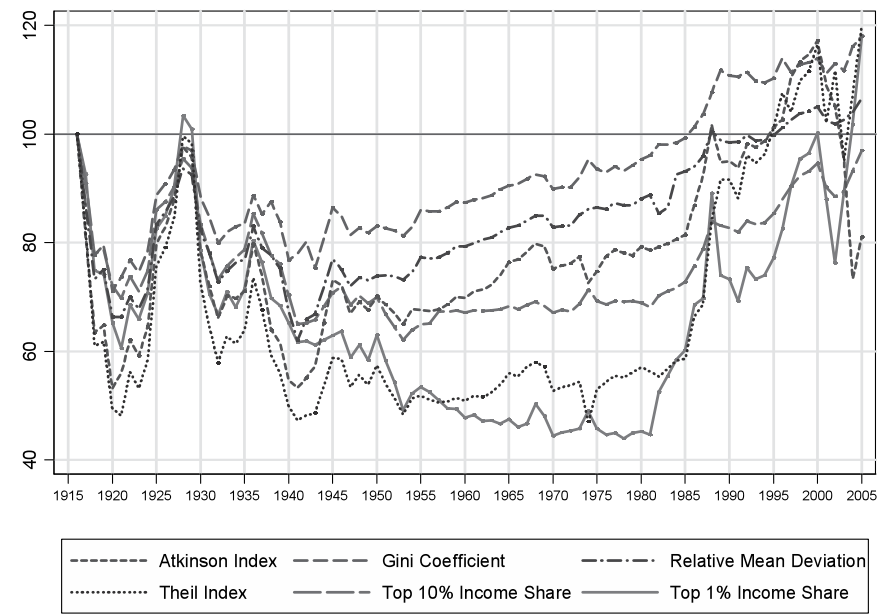


The relative mean deviation can be defined as representing the average absolute distance between each person's income and the mean income of the population. It varies between zero and two, with larger values indicating higher inequality. Unlike the other three measures, the relative mean deviation fails to satisfy even the weak principle of transfers, meaning it is possible to have a reallocation of income without an associated change in inequality. Over our sample period, the relative mean deviation has a mean and standard deviation of 0.66 and 0.11 , with a range of annual averages between 0.49 and 0.84 (occurring in 1941 and 2005, respectively). Table 4 shows that the evolution of the relative mean deviation over the sample is closely correlated with both the Atkinson index and Gini coefficient.

The Gini coefficient can be defined as representing the average distance between all pairs of proportional income in the population. It varies between zero and one, with higher values indicating greater inequality, and is known for being sensitive to transfers in the middle of the income distribution (Cowell 1995, p.23). The Gini coefficient satisfies the weak principle of transfers, meaning any reallocation of income will be associated with a change in overall inequality. Like the relative mean deviation, however, the Gini coefficient has the unattractive property of being non-decomposable. Hence, it is possible for each subgroup in the population to experience an increase in inequality, while overall inequality shows a decrease. This property makes it difficult to disaggregate changes in overall inequality into changes among specific subgroups of the population. Over our sample period, the Gini coefficient has a mean value of 0.47 , with a standard deviation of 0.07 . As Table 4 shows, it is most closely correlated with the relative mean deviation.

The Atkinson index of inequality is a social welfare function based measured of inequality bound between zero and one, with higher values indicating greater inequality. It is analytically appealing since it is both decomposable and satisfies the weak principle of transfers. The Atkinson measure we employ uses an inequality aversion parameter $(\boldsymbol{\varepsilon})$ of 0.5 , meaning the index is more sensitive to changes in the upper-end of the income distribution. The mean and standard deviation for the Atkinson index over our sample period is 0.19 and 0.05 , with a range of annual averages between 0.13 and 0.28 (occurring in 1920 and 2000, respectively). 
Table 4

Correlations of the Inequality Measures

\begin{tabular}{|l|c|c|c|c|c|c|}
\hline & $\begin{array}{c}\text { Atkinson } \\
\text { Index }\end{array}$ & $\begin{array}{c}\text { Gini } \\
\text { Coefficient }\end{array}$ & $\begin{array}{c}\text { Relative } \\
\text { Mean } \\
\text { Deviation }\end{array}$ & $\begin{array}{c}\text { Theil } \\
\text { Entropy } \\
\text { Index }\end{array}$ & $\begin{array}{c}\text { Top 10\% } \\
\text { Income } \\
\text { Share }\end{array}$ & $\begin{array}{c}\text { Top 1\% } \\
\text { Income } \\
\text { Share }\end{array}$ \\
\hline $\begin{array}{l}\text { Atkinson } \\
\text { Index }\end{array}$ & 1.000 & - & - & - & - & - \\
\hline $\begin{array}{l}\text { Gini Coef- } \\
\text { ficient }\end{array}$ & 0.875 & 1.000 & - & - & - & - \\
\hline $\begin{array}{l}\text { Relative } \\
\text { Mean } \\
\text { Deviation }\end{array}$ & 0.915 & 0.975 & 1.000 & - & - & - \\
\hline $\begin{array}{l}\text { Theil } \\
\text { Entropy } \\
\text { Index }\end{array}$ & 0.905 & 0.798 & 0.829 & 1.000 & - & - \\
\hline $\begin{array}{l}\text { Top 10\% } \\
\text { Income } \\
\text { Share }\end{array}$ & 0.838 & 0.741 & 0.794 & 0.924 & 1.000 & - \\
\hline $\begin{array}{l}\text { Top 1\% } \\
\text { Income } \\
\text { Share }\end{array}$ & 0.729 & 0.604 & 0.642 & 0.886 & 0.926 & 1.000 \\
\hline
\end{tabular}

It is apparent from Figure 6 that the Gini coefficient, and to a lesser extent the relative mean deviation and Atkinson index, portray two pronounced differences over the last ninety years when compared with the two top income shares. First, the decrease in inequality after the Great Depression and World War II is not as precipitous. Second, the Gini coefficient surpasses its pre-Great Depression high in 1985, more than a decade before either of the two top income share measures.

One possible explanation for these relative differences is that inequality in the upper-end of the income distribution fell considerably more at mid-century. Hence, the smaller decline in the Atkinson index, Gini coefficient, and relative mean deviation are meaningful differences driven by the broad nature of inequality captured by these three measures. The top $1 \%$ and $10 \%$ income shares, by contrast, are not broad distributional measures, and thus portray meaningful distinctions. Alternatively, it is plausible that the additional measures are simply inefficient measures of inequality in the context of IRS income data, since IRS data is truncated below a threshold level of income, as discussed in the previous section. ${ }^{10}$ 
Table 4 shows that the top 10\% income share is most closely correlated with the fourth and final additional measure, Theil's entropy index. The Theil index is an unbound derivative of statistical information theory where larger values indicate greater income inequality. It is both decomposable and, unlike the other inequality measures, satisfies the strong principle of transfers. The latter is exclusive to generalized entropy indexes, and implies that changes in inequality from reallocations of income depend only on the relative distances between individuals, not their locations within the overall distribution. In Figure 6, the Theil index appears to closely follow the trend of the top $1 \%$ share of income, with high levels of income inequality at the beginning and end of the sample, and low levels of inequality between 1940 and 1980. Over the sample period, the mean and standard deviation for the Theil index are 0.49 and 0.21 , with an annual average range of 0.34 to 0.86 (in 1974 and 2005, respectively).

\section{CONCLUSIONS}

For many U.S. states the income share of top income earners has experienced a distinct U-shaped pattern over the last century. Following early-century peaks in measures of income inequality, inequality declined substantially during the Great Depression and World War II. The lowest level of income inequality for many states occurred during the 1950s. After decades of post-World War II stability, however, large increases in income inequality began again in the 1980s, with a significant part of this increase occurring after the Tax Reform Act of 1986, and continuing throughout the 1990s. There appears to be significant state-level variations, however, both before the year 1945, and regionally. While Northeastern states appear most closely associated with overall U.S. trends, Western states show the least amount of association.

The dual peak in income inequality before the Great Depression and again during the new millennium raises important economic, political, and sociological questions. This paper seeks to contribute to these discussions by providing a comprehensive state-level panel of annual income inequality measures covering the ninety year period 1916 to 2005 . Recent empirical research on income inequality has usually relied on the aggregate U.S. time-series data of Piketty and Saez (2003), or the low-frequency cross-national panel of Deininger and Squire (1996). State-lev- 
el panels, though underutilized relative to international panels, can be constructed in low-frequency form using data from the decennial census (e.g., Partridge 1997, 2005), or for more recent years using the March Current Population Survey (e.g., Doyle, Ahmed, and Horn 1999).

Important caveats arise with our new panel, however, since our measures of inequality are constructed from individual tax filing data available from the Internal Revenue Service. Although IRS income data is the only informational source available annually for each state since 1916, it does not include income on some individuals at the low-end of the income distribution. The censoring threshold varies by age, marital status, and most notably, tax filing year. As a consequence, our data appears best suited for assessing changes in the upper-end of the income distribution.

Used in conjunction with other existing data, it is hoped that the availability of our ninety year annual state-level panel can further illuminate both the causes and consequences of the income inequality changes over the last century. For example, an early version of this data set covering the period 1945-2004 was used to explore the association of income inequality on economic growth during the post-war period (see Frank 2009a and 2009b). Clearly the large and balanced size of our new panel offers several potential advantages in the furthering empirical research surrounding income inequality. By combining variation across states with the variation over time, a state-level panel offers less-collinearity, a greater ability to control for unobserved heterogeneity, and improvements the efficiency of the econometric estimates over strictly time-series data. The greater number of observations in the panel increases the degrees of freedom, and allows for the testing of more complicated econometric models. Moreover, following of the same states over time permits one to control for both state-invariant and time-invariant variables, and better enables the study of dynamics and speed of adjustment than either purely time-series or cross-sectional data. Finally, following the same states over a long period permits exogenous variation in policies and institutions, and facilitates the identification of parameters of interest.

\section{APPENDIX - CONSTRUCTION OF THE INEQUALITY MEASURES}

Since IRS income data is reported in income groups, the percentile ranking measures are based on the split histogram interpolation method proposed by Cowell (1995), whereby the proportion of the sample population with income less than or 
equal to income $y$ is defined as

$$
F(y)=F_{i}+\int_{a_{i}}^{y} \varphi_{i}(x) \mathrm{d} x,
$$

where $a_{i}$ is the lower bound of group $i$, and $F_{i}$ is the cumulative frequency of the number of individuals before group $i{ }^{11}$ The proportion of the total income received by those with income less than or equal to is given by

$$
\phi(y)=\phi_{i}+\frac{1}{\mu} \int_{a_{i}}^{y} x \varphi_{i}(x) \mathrm{d} x,
$$

where $\mu$ is mean income. The density within each interval $i$ is defined by the split histogram density:

$$
\varphi_{i}=\left\{\begin{array}{l}
\frac{f_{i}\left(a_{i+1}-\mu_{i}\right)}{\left(a_{i+1}-a_{i}\right)\left(\mu_{i}-a_{i}\right)}, \text { for } a_{i} \leq x \leq \mu_{i} \\
\frac{f_{i}\left(u_{i}-a_{i}\right)}{\left(a_{i+1}-a_{i}\right)\left(a_{i+1}-\mu_{i}\right)}, \text { for } \mu_{i} \leq x \leq a_{i+1}
\end{array}\right.
$$

where $f_{i}$ is the relative frequency of $n_{i}$ within group $i$, and $a_{i+1}$ is the upper bound of group $i .^{12}$

The Gini coefficient we construct is the compromise Gini coefficient proposed by Cowell and Mehta (1982) and Cowell (1995). Accordingly, the lower limit of the Gini can be derived based on the assumption that all individuals in a group receive exactly the mean income of the group:

$G_{L}=\frac{1}{2} \sum_{i=1}^{k} \sum_{j=1}^{k} \frac{n_{i} n_{j}}{n \mu}\left|\mu_{i}-\mu_{j}\right|$

where $\mathrm{n}$ is the number of individuals, and subscripts $\mathrm{i}$ and $\mathrm{j}$ denote within group values. The upper limit Gini can be constructed based on the assumption that individuals within the group receive income equal to either the lower or upper bound of the group interval:

$G_{U}=G_{L}+\sum_{i=1}^{k} \frac{n_{i}^{2}\left(a_{i+1}-\mu_{i}\right)\left(\mu_{i}-a_{i}\right)}{n_{i}^{2} \mu\left(a_{i+1}-a_{i}\right)}$.

Given equations (4) and (5), the compromise Gini coefficient proposed by Cowell and Mehta (1982) is simply: $\mathrm{G}_{\mathrm{U}} 2 / 3+\mathrm{G}_{\mathrm{L}} 1 / 3$. 
The remaining three measures can be derived using the general form:

$J=\sum_{i=1}^{k} \int_{a_{i}}^{a_{i}+1} h(y) \varphi_{i}(y) \mathrm{d} y$,

where $\varphi_{i}^{i}{ }_{i}{ }_{i}$.is the split histogram density, and $h(y)$ is an evaluation function. To construct the Atkinson index, the evaluation function is defined as:

$h(y)=\left(\frac{y}{\mu}\right)^{1-\varepsilon}$

where $1-J^{1 / 1-\varepsilon}$. Note that $\varepsilon$ is the Atkinson inequality aversion parameter. The evaluation function for the relative mean deviation is defined as:

$h(y)=\left|\frac{y}{\bar{y}}-1\right|$

To construct the Theil entropy index, the evaluation function is defined as:

$h(y)=\frac{y}{\mu} \ln \left(\frac{y}{\mu}\right)$

Unlike the percentile rankings, Gini coefficient, or relative mean deviation, the Atkinson index and Theil index are undefined for negative incomes. Hence, to construct the Atkinson and Theil inequality measures, negative IRS income data must be truncated, meaning the lowest possible income, $a_{1}$, is $\$ 0$.

\section{BIOGRAPHICAL SKETCH OF AUTHOR}

Mark Frank is a Professor of Economics and International Business at Sam Houston State University. He received his Ph.D. from the University of Texas at Dallas. His research interests include telecommunications regulation, the economics of advertising in the liquor industry, and income inequality in the United States.

\section{REFERENCES}

Akhand, H., \& Liu, H. (2002). Income Inequality in the United States: What the Individual Tax Files Say. Applied Economics Letters, 9(4), 255-259.

Baltagi, B.H. (2005). Econometric Analysis of Panel Data/ Badi H. Baltagi. New York, N.Y.: John Wiley \& Sons.

Barro, R.J. (2000). Inequality and Growth in a Panel of Countries. Journal of Economic Growth, 5(1), 5-32.

Cowell, F.A. (1995). Measuring inequality, (2nd Ed.). New York and Toronto, N.Y.: 
Simon and Schuster International, Harvester Wheatsheaf/Prentice Hall, London Cowell, F.A., \& Mehta, F. (1982). The Estimation and Interpolation of Inequality Measures. Review of Economic Studies, 49(2), 273-290.

Deininger, K., \& Squire, L. (1996). A New Data Set Measuring Income Inequality. World Bank Economic Review, 10(3), 565-591.

Doyle, J.M., Ahmed, E. \& Horn, R.N. (1999). The Effects of Labor Markets and Income Inequality on Crime: Evidence from Panel Data. Southern Economic Journal, 65(4), 717-738.

Frank, M. W. (2009). Inequality and Growth in the United States: Evidence from a New State-Level Panel of Income Inequality Measures. Economic Inquiry, 27(1), 55-68.

Frank, M. W. (2009). Income Inequality, Human Capital, and Income Growth: Evidence from a State-Level VAR Analysis. Atlantic Economic Journal, 37(2), 173-185.

Goldin, C., \& Margo, R.A. (1992). The Great Compression: The Wage Structure in the United States at Mid-century. Quarterly Journal of Economics, 107(1), 1-34. Gottschalk, P. (1997). Inequality, Income Growth, and Mobility: The Basic Facts. Journal of Economic Perspectives, 11(2), 21-40.

Hollenbeck, S., \& Kahr, M.K. (2008). Ninety Years of Individual Income Tax Statistics, 1916-2005. Statistics of Income Bulletin, 27(3), 136-147.

Hsiao, C. (2003). Analysis of panel data. (2nd Ed.). Cambridge University Press.

Krueger, A.B. (2003). Inequality, Too Much of a Good Thing. Inequality in America:

What role for human capital policies?, eds. J.J. Heckman \& A.B. Krueger, MIT Press, Cambridge, 1-75.

Levy, F., \& Murnane, R.J. (1992). U.S. Earnings Levels and Earnings Inequality: A Review of Recent Trends and Proposed Explanations. Journal of Economic Literature, 30(3), 1333-1381.

Li, H., Squire, L., \& Zou, H. (1998). Explaining International and Intertemporal Variations in Income Inequality. Economic Journal, 108(446), 26-43.

Morgan, J. (1962). The Anatomy of Income Distribution. The Review Of Economics And Statistics, 44(3), 270-283.

Partridge, M.D. (2005). Does Income Distribution Affect U.S. State Economic Growth?. Journal of Regional Science, 45(2), 363-394.

Partridge, M.D. (1997). Is Inequality Harmful for Growth? Comment. American Economic Review, 87(5), 1019-1032. 
Pesaran, M.H., Shin, Y. \& Smith, R.P. (1999). Pooled Mean Group Estimation of Dynamic Heterogeneous Panels. Journal of the American Statistical Association, 94(446), 621-634.

Pesaran, M.H. \& Smith, R. (1995). Estimating Long-Run Relationships from Dynamic Heterogeneous Panels. Journal of Econometrics, 68(1), 79-113.

Phillips, P.C.B. \& Moon, H.R. (2000). Nonstationary Panel Data Analysis: An Overview of Some Recent Developments. Econometric Reviews, 19(3), 263-286.

Phillips, P.C.B. \& Moon, H.R. (1999). Linear Regression Limit Theory for Nonstationary Panel Data. Econometrica, 67(5), 1057-1111.

Piketty, T. \& Saez, E. (2003). Income Inequality in the United States, 1913-1998. Quarterly Journal of Economics, 118(1), 1-39.

Quah, D. (2001). Some Simple Arithmetic on How Income Inequality and Economic Growth Matter, Working Paper Edition, London School of Economics. 